\title{
Assessment of varicella vaccine effectiveness in Germany: a time-series approach
}

\author{
M. HÖHLE ${ }^{1,2 *}$, A. SIEDLER ${ }^{1}$, H.-M. BADER ${ }^{3}$, M. LUDWIG ${ }^{4}$, U. HEININGER $^{5}$ \\ AND R. VON KRIES \\ ${ }^{1}$ Department for Infectious Disease Epidemiology, Robert Koch Institute, Berlin, Germany \\ ${ }^{2}$ Department of Statistics, Ludwig-Maximilians-University München, Munich, Germany \\ ${ }^{3}$ Working Vaccination Committee, Ministry for Health, Schleswig-Holstein, Germany \\ ${ }^{4}$ Kassenärztliche Vereinigung Schleswig-Holstein, Germany \\ ${ }^{5}$ University Children's Hospital Basel, Basel, Switzerland \\ ${ }^{6}$ Institute of Social Paediatrics and Adolescent Medicine, Ludwig-Maximilians-University München, \\ Munich, Germany
}

(Accepted 9 November 2010; first published online 15 December 2010)

\section{SUMMARY}

A multivariate time-series regression model was developed in order to describe the 2005-2008 age-specific time-course of varicella sentinel surveillance data following the introduction of a varicella childhood vaccination programme in Germany. This ecological approach allows the assessment of vaccine effectiveness under field conditions by relating vaccine coverage in cohorts of 24-month-old children to the mean number of cases per reporting unit in the sentinel network. For the 1-2 years age group, which is directly affected by the vaccination programme, a one-dose vaccine effectiveness of $83 \cdot 2 \%(95 \%$ CI $80 \cdot 2-85 \cdot 7)$ was estimated which corresponds to previous approaches assessing varicella vaccine effectiveness in the field in the USA.

Key words: GP surveillance systems, infectious disease epidemiology, statistics, vaccines, varicella zoster.

\section{INTRODUCTION}

Varicella vaccination programmes have been implemented in several countries during the last decade [1-4]. Evaluations of the varicella vaccination programme in the USA have demonstrated an impressive effect with respect to varicella-related mortality [1], hospitalization [2, 3] and incidence [4], despite limited vaccine efficacy of one dose [5] and even two doses of varicella vaccine $[6,7]$. However, the

* Author for correspondence: Dr M. Höhle, Department for Infectious Disease Epidemiology, Robert Koch Institute, DGZ-Ring 1, 13086 Berlin, Germany.

(Email: hoehlem@rki.de) epidemiological characteristics for the spread of varicella in unvaccinated populations vary considerably [8] making assessment of the effects of vaccination programmes on a national level mandatory.

Since varicella is very common in early childhood [9] assessment of the incidence of temporal trends of varicella incidence at a national level is not feasible. Therefore trends are either monitored regarding incidence in regional samples $[1,8]$ or in sentinel surveys [10]. While trends in case ascertainment can be depicted by these approaches, quantifying vaccine effects and age-group interdependencies may require a more sophisticated approach. We therefore attempted to model the time-course of varicella following the 
introduction of a universal childhood vaccination programme in Germany in 2004 [10] targeted at children aged 1-2 years and sought to apply these analyses to an ecological approach in order to assess varicella vaccine effectiveness.

Measuring the success of an immunization programme under field conditions is a particular challenge which can be addressed by epidemiological means. The study of vaccine effectiveness typically investigates direct effects in individuals using different vaccination models and study designs [11]. However, the literature on quantifying population effects of vaccination is less developed and typically occurs within the framework of a detailed dynamic model for disease transmission (see e.g. [12, 13] for such an approach in the context of varicella vaccination). However, for data originating from routine monitoring such models are too detailed. The screening method [14] is one approach operating under such monitoring conditions, but it requires knowledge about both the vaccination status of cases and the proportion of population vaccinated, which are not always available. Furthermore, the approach does not specifically address the time-varying aspect of coverage following the introduction of a vaccination programme. Our selected ecological approach provides a solution for estimating population-level effects of vaccination under field conditions by using a coarse disease transmission model based on time-series methodology which indirectly takes herd immunity effects into account. Classical time-series modelling has already received some attention in analysing vaccine effects in populations [15-19]. However, previous analyses are typically restricted to univariate analyses with information on vaccination coverage entered only in binary form. Our approach continues along these lines and is novel in as much as it considers coverage percentage as an exogenous time series and, being a multivariate time-series model, handles progression in different age strata.

Given the above, our research question can be formulated as follows: does modelling the time-course of varicella following the introduction of the varicella vaccination programme in Germany allow for quantification of age-specific effects and for an ecological assessment of vaccine effectiveness? Specifically, we wanted to quantify direct vaccine effects in the vaccinated $1-2$ years age group by using seasonal and autoregressive terms in the model as proxy for adjusting for indirect vaccine effects in all regarded age groups.

\section{METHODS}

\section{Data sources}

A convenience sample of 1176 primary-care physicians, consisting of $57 \%$ paediatricians and $43 \%$ general practitioners (GPs) which accounted for about $15 \%$ of all German paediatricians and about $1 \%$ of all German GPs in private practice was the sampling frame for the sentinel network. Sentinel network physicians of both groups are distributed in German federal states in the same proportion as the total number of respective physicians in private practice. Physicians reported aggregated monthly numbers of varicella cases by age group. Clear case definitions for reporting were provided. A case of varicella is defined as a person presenting with a clinical picture compatible with varicella, i.e. the presence of skin exanthema and concomitant presentation of papules, blisters, pustules, crusts [10]. Zeroreporting and active reminders were included, but as physicians participated voluntarily without any incentives for their reports, the additional workload for them needed be kept as low as possible. Hence, vaccination status of cases was not recorded and only aggregated case numbers by age groups were reported. Furthermore, as patients are free to choose and change their physician and only a sample of physicians report to the sentinel network, the population size under surveillance can not be defined [10].

Vaccine uptake was extrapolated from health insurance data in one [Schleswig-Holstein $(\mathrm{SH})]$ of the 16 German federal states. Vaccination billing for about $90 \%$ of all children in Germany is handled by the Association of Statutory Health Insurance Physicians ('Kassenärztliche Vereinigungen') in the different German federal states [20]. Children in the health insurance system were traced from birth to age 24 months upon billing for the well-baby check visits. Varicella vaccination status by age 24 months was assessed for consecutive birth cohorts from 2003 to 2006 and this constituted the numerator for assessing vaccination coverage for the different birth cohorts.

\section{Analyses}

Data from all paediatricians and GPs participating in the sentinel network were used in the analysis. As response for the time-series modelling we chose the monthly 'mean number of varicella cases per reporting unit'. This quantity accounts for the varying number of reporting units in the sentinel network, 


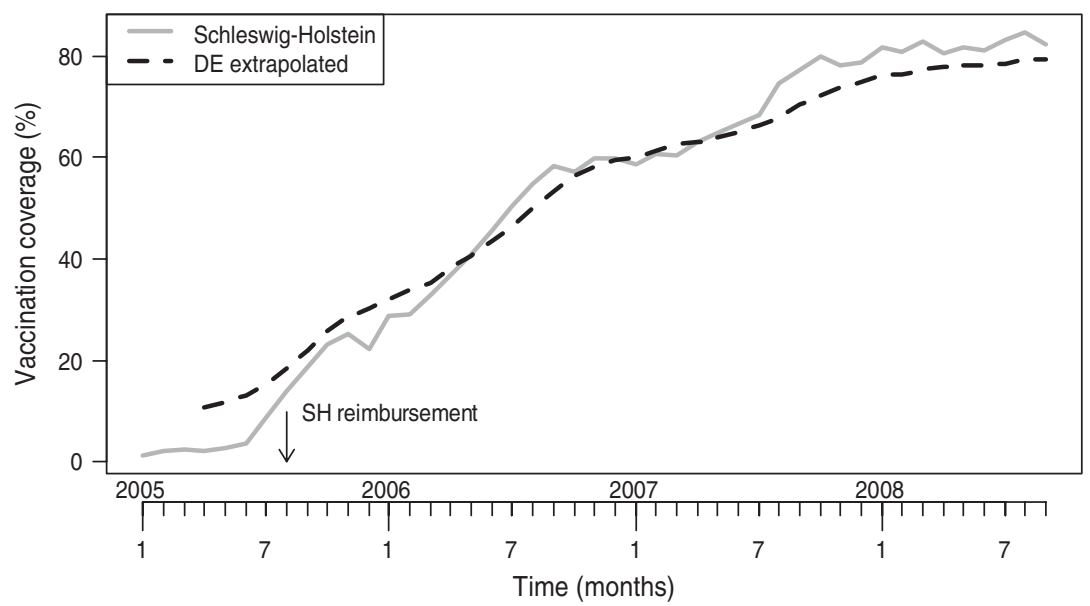

Fig. 1. Vaccination coverage in the 24-month-old birth cohort in Schleswig-Holstein ( $\mathrm{SH}$ ) and the extrapolated coverage for Germany.

whereas the size of the reporting units is considered to be stable over time. The dynamics of the response variable over time is described by a two-phase model: at the first level the mean number of varicella cases at time $t$ (measured in months) per sentinel reporting unit was modelled. This was done using a linear predictor with intercept, linear trend, seasonal terms and an additional $\operatorname{ARMA}(p, q)$ model with $p$ autoregressive (AR) and $q$ moving-average (MA) terms. At the second level the age distribution of the observed cases at time $t$ in the $<1,1-2,3-4,5-9$ and $>9$ years age groups was handled by a multinomial logistic regression model (for further details on the modelling see the Appendix). For levels 1 and 2, Akaike's Information Criterion (AIC) was used to select from a set of candidate models. A strong correlation between vaccination coverage and time (measured in months) was expected. Including both month and vaccination coverage as covariables would thus lead to strong collinearity and hence lead to stability problems if they are both included. Therefore, the following strategy was applied: fit separate models including either month or coverage as covariate and use AIC to select the better model.

We used R [21] - a free software environment for statistical computing and graphics - to perform all statistical computations.

In order to estimate vaccination coverage nationally, coverage estimates for $\mathrm{SH}$ were extrapolated to the whole of Germany as follows. For each of the 16 federal states the SH time-series was shifted in time such that the time of reimbursement in $\mathrm{SH}$ (August 2005) was moved to the corresponding month of reimbursement in the federal state. The resulting 16 time series were then averaged by weighting with the number of sentinel network units for the respective federal state in that month.

Vaccine effectiveness is conventionally defined as the reduction between the risk of unvaccinated and vaccinated individuals divided by the risk of unvaccinated individuals [22], i.e. 1 minus the relative risk. In our model we use a before-and-after approach to estimate vaccine effectiveness under field conditions. We consider $0 \%$ coverage as baseline - representing the unvaccinated situation - and compare this with $100 \%$ coverage, corresponding to the vaccinated situation. Using this approach, vaccine effectiveness can be estimated in the unstratified situation as 1 minus the relative reduction in the expectation of the response, i.e. $1-\mu_{100} \%$ coverage $/ \mu_{0} \%$ coverage $=1-\exp \left(\beta_{1}\right)^{100}$, where $\beta_{1}$ represents the effect of coverage in the level 1 model described in the Appendix. Since coverage is only available for the 1-2 years age group, we obtained an estimate of vaccine effectiveness for this specific age group by computing the relative reduction in expectation for this age group, i.e. $1-\mu_{100} \%$ coverage,1-2 $\mathrm{yr} /$ $\mu_{0} \%$ coverage, $1-2$ yr (see Appendix for details on how these expectations are obtained by a combination of level 1 and level 2 models).

\section{RESULTS}

Figure 1 shows the monthly vaccination coverage among the 24-month-old birth cohort in SH. Here, reimbursement started in August 2005. Also shown in Figure 1 is our extrapolated national coverage timeseries; the extrapolated coverage percentage at the beginning of the sentinel network in April 2005 was 


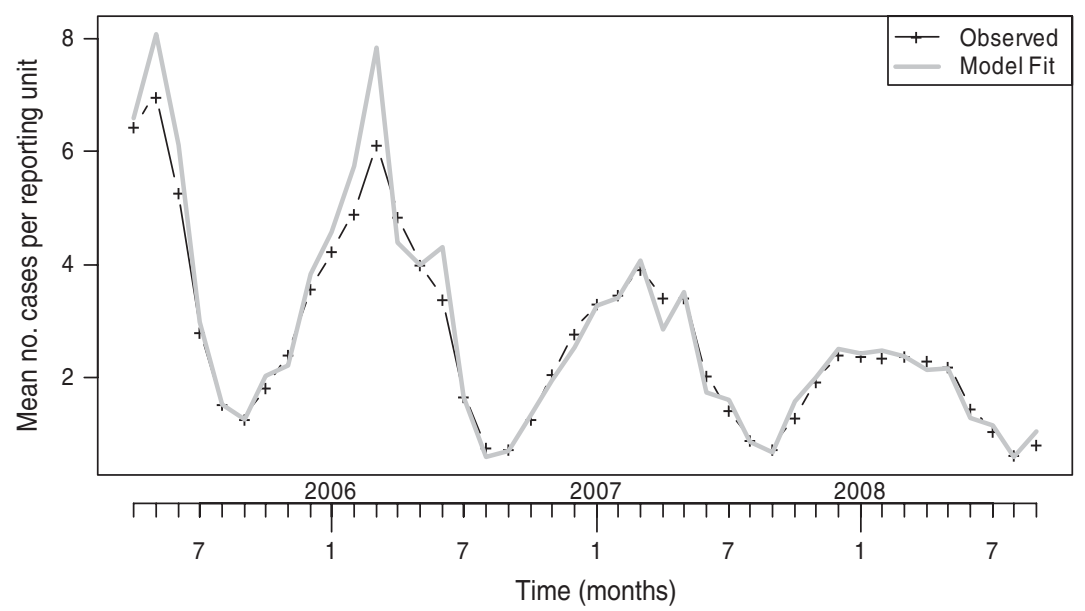

Fig. 2. Observed and model-fitted mean number of varicella cases per reporting unit in the sentinel network.

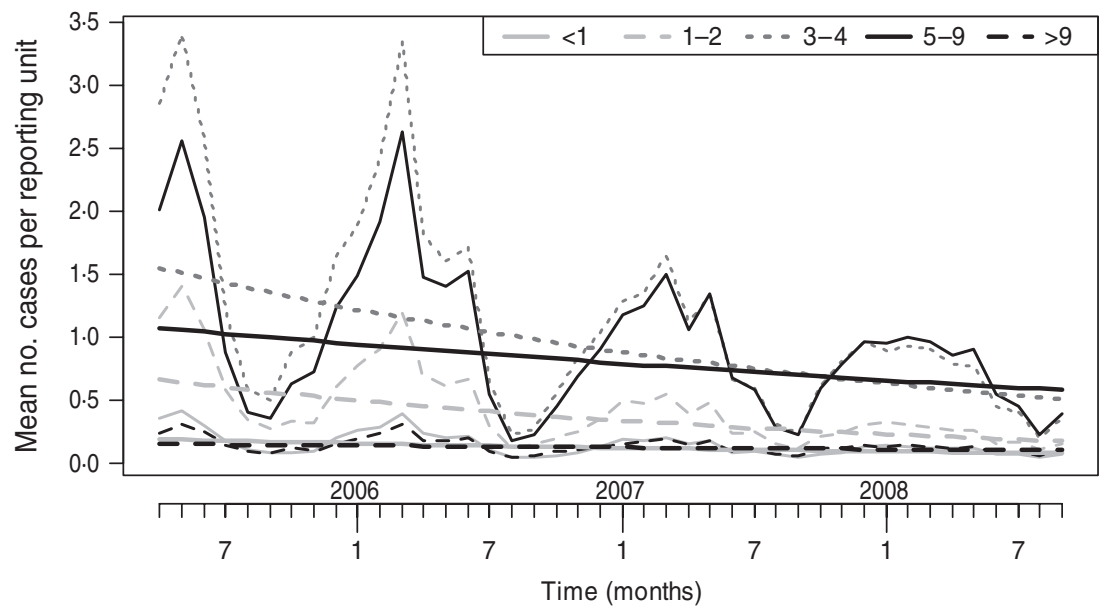

Fig. 3. Two-level model-fitted mean number of cases for each of the five age groups. Also shown are the trends $\mu_{t, i}$ (smooth lines) for each age group $i$ and time-point $t$ obtained by combining the intercept and trend components of the level 1 and level 2 models.

$11 \%$. The corresponding coverages were $38 \%, 63 \%$, $78 \%$, respectively, at 1,2 and 3 years after the sentinel network started. A rough comparison (not shown) with differently collected yearly coverage rates for the 24-month-olds from the 2004 and 2005 birth cohorts in an available subset of ten federal states showed that our extrapolation provided a fair description of the coverage of these two cohorts.

The mean number of monthly reporting units in the sentinel network for the available 42 months of monitoring was 679 (s.D. $=41$ ). Figure 2 shows the observed monthly time series of mean number of cases per reporting unit together with the fit of our level 1 model containing intercept, trend and periodic function, $f(t)$, consisting of two harmonics with frequencies of 12 and 6 months, respectively. An
$\operatorname{ARMA}(2,1)$ model captured the remaining autocorrelation of the error terms. This level 1 model was determined as the best-fitting model according to AIC in the investigated set of candidate models. The estimated coefficient for the time trend in this model is $-0.023(95 \% \mathrm{CI}-0.025$ to -0.020$)$, i.e. the reduction in the mean number of cases per reporting unit is significant $(P<0.001)$ and corresponds to a decrease in the mean number of cases per reporting unit by a factor of $\exp (-0.023)=0.978$ per month or $\exp (-0.023 \times 12)=0.762$ per year.

Figure 3 shows the model-fitted values combining level 1 and level 2 models (using month as linear covariate). Also shown are the estimated time trends, $\mu_{t, i}$, for each of the five age groups $(<1,1-2,3-4,5-9$, $>9$ years) as described in the Appendix. 
Table 1. Model-predicted relative reduction factors for each of the five age groups

\begin{tabular}{llllll}
\hline \hline \multirow{5}{*}{ Ratio } & \multicolumn{5}{l}{ Age group (years) } \\
\cline { 2 - 6 } & $<1$ & $1-2$ & $3-4$ & $5-9$ & $>9$ \\
\hline$\mu_{13, i} / \mu_{1, i}$ & 0.77 & 0.68 & 0.73 & 0.84 & 0.89 \\
$\mu_{25, i} / \mu_{13, i}$ & 0.76 & 0.68 & 0.72 & 0.84 & 0.89 \\
$\mu_{37, i} / \mu_{25, i}$ & 0.76 & 0.67 & 0.72 & 0.83 & 0.88 \\
\hline \hline
\end{tabular}

A decline in all five age groups was observed. For better interpretation, Table 1 shows the modelpredicted yearly relative reductions for the first three full years of the sentinel network. As expected, the highest decline is observed in the 1-2 years age group, but all other age groups also experienced a decline. For example, the percentage reduction over 3 years in the $<1$ year age group is given by $1-0.77 \times 0.76 \times$ $0 \cdot 76=56 \%$. The corresponding reductions in the other groups were $69 \%, 62 \%, 42 \%$ and $30 \%$. Since no vaccinations were performed in the $<1$ year age group, the reduction here may be entirely due to herd effect.

However, the extrapolated national vaccination coverage percentage shown in Figure 1 has a Pearson correlation coefficient with month of 0.97. Using either month or coverage percentage in the model shows that the model with the covariate coverage provides the better fit (AIC of $-44.66 v s$. $-39 \cdot 73$ ). When replacing the term $\beta_{1} t$ with $\beta_{1}$ (coverage in $\%$ ) in the level 1 model, the estimated coefficient of coverage is $-0.013(95 \% \mathrm{CI}-0.014$ to -0.011$)$, which means that the coverage effect is significant $(P<0 \cdot 001)$ and for each percentage point of vaccination coverage in the 24-month-old cohort the mean number of cases per reporting unit is reduced by a factor of 0.987 . While this approach describes the overall impact of the vaccination programme, the assessment of vaccine effectiveness requires confining the analysis to vaccinated age groups only.

To specifically target the reduction in the $1-2$ years age group, for whom the vaccine uptake could be estimated and who experience the direct vaccine effects, we combined the above findings with the level 2 model where the linear time trend is also replaced with coverage, i.e. coverage is used as single linear covariate in both level 1 and level 2 models, but the harmonic functions at both levels remain functions of time. This allows us to predict the reduction factor such as $\mu_{100 \%, 1-2 \mathrm{yr}} / \mu_{0 \%, 1-2 \mathrm{yr}}$ for the 1-2 years age group, i.e. the reduction of a hypothetical $100 \%$
Table 2. Model-predicted reduction factors for five hypothetical coverage percentages

\begin{tabular}{lll}
\hline \hline & \multicolumn{2}{l}{ Predicted reduction factor } \\
\cline { 2 - 3 } Coverage & $\mu_{\text {coverage } \%, 1-2 \text { yr } / \mu_{0} \%, 1-2 \text { yr }}$ & $95 \%$ CI \\
\hline $60 \%$ & $0 \cdot 349$ & $0 \cdot 317-0 \cdot 384$ \\
$70 \%$ & $0 \cdot 291$ & $0 \cdot 260-0 \cdot 326$ \\
$80 \%$ & $0 \cdot 243$ & $0 \cdot 214-0 \cdot 277$ \\
$90 \%$ & $0 \cdot 202$ & $0 \cdot 175-0 \cdot 235$ \\
$100 \%$ & $0 \cdot 168$ & $0 \cdot 143-0 \cdot 198$ \\
\hline \hline
\end{tabular}

CI, Confidence interval.

vaccination coverage in the 24-month-old birth cohort compared to a coverage baseline of $0 \%$. This comparison mimics the conventional vaccine effectiveness assessment comparing attack rates in vaccinated and unvaccinated individuals. Table 2 gives the results for coverage percentages between $60 \%$ and $100 \%$. This yields a vaccine effectiveness of $83 \cdot 2 \%$ $(95 \%$ CI $80 \cdot 2-85 \cdot 7)$ in the $1-2$ years age group.

\section{DISCUSSION}

Since the implementation of most vaccination programmes is progressive with gradual increments of vaccination coverage, the decrease of the targeted diseases due to direct and indirect vaccine effects will also be progressive. During the implementation of a universal varicella childhood vaccination programme in Germany we demonstrated that modelling of the time-course of disease allows estimation of the reduction for different age groups for comparison of direct and indirect vaccination effects. With further inclusion of vaccine uptake, an ecological estimate for vaccine effectiveness was possible and yielded almost identical results to other varicella vaccine effectiveness estimates.

Although year-to-year variation in the incidence of varicella (measured by GP visits or hospitalizations) has been reported from various countries and settings, the predicted and observed decline of varicella cases in the German sentinel network system, mainly in the 1-2 years age group, is consistent with an effect of vaccination rather than a secular trend. Given the short time-span of the monitoring period it appears unlikely that the reduction is attributable to secular changes in factors other than vaccine use [19]. Other epidemiological investigations and modelling approaches [23-26] also indicate a lack of large 
multi-year periodicities. However, our approach remains sensitive to unexplained trends in incidence, whereas ordinary year-to-year fluctuations are taken into account by the stochastic nature of the model.

Early detection of a herd immunity effect in children aged $<1$ year and the finding of similar vaccine effectiveness estimates for the ecological compared to other approaches are important findings with respect to varicella vaccination programmes.

Impressive herd immunity effects of varicella vaccination programmes have previously been shown in adults, e.g. a reduction of $74 \%$ over a 10 -year period with increasing childhood vaccination coverage in the USA [27]. Even more impressive reductions than in adults or adolescents have been reported in infants too young to be immunized themselves $[1,15,16]$. These impressive indirect effects were achieved over a 6-year period with $>90 \%$ coverage since programme implementation [28] and over 10 years with vaccination coverage $>80 \%$ achieved after the fifth year [29]. Our results show, that even with coverage $<80 \%$ first effects of indirect protection might occur.

Scientifically more relevant, however, might be the suggested ecological approach to estimate vaccine effectiveness based on time-series methodology. Using vaccine uptake in the model instead of time to estimate the reduction of reported varicella cases per increment in vaccine uptake appears justified because the model fit for vaccine uptake was considerably better than the fit for year of observation. A hypothetical vaccine uptake of $100 \%$ would reflect the effectiveness of the vaccine in vaccinated individuals. Vaccination status at age 24 months was used as an estimate for the 1-2 years age group, which covers children aged 12-35 months. We expected that choosing coverage at the midpoint of the age group balances between lower and higher coverage rates in children aged 12-23 and 24-35 months. The reduction of the reported varicella cases for each $1 \%$ increment in vaccination coverage was used to estimate vaccine effectiveness from an ecological approach. As the time-series model contains autoregressive terms, herd immunity effects are indirectly addressed in the modelling. A decrease in the mean number of cases (and hence the infection pressure) during previous months implies a smaller contribution from the past for the current response.

Estimates of varicella vaccine effectiveness vary depending on the setting of the assessment [30], severity of the infection [5], number of vaccine doses [6] and duration of follow-up [31]. Most of these were based on outbreak investigations [30, 32], a cohort study [33] a household contact study [34] or a casecontrol study [35]. The median effectiveness has been estimated at $72 \%$ and $71 \%[30,32]$ for all and $96 \cdot 5 \%$ or $89 \%[5,30]$, respectively, for moderate to severe infections from outbreak investigations. The respective figures were $83 \%$ and $100 \%$ in a prospective cohort study [33], $85 \%$ and $97 \%$ in a case-control study confined to laboratory-confirmed cases [35] and 79\% and $92 \%$ in a household contact study for moderate cases [34]. The German varicella childhood vaccination programme was initiated with one-dose varicella vaccination and only recently switched to a two-dose schedule in 2009. The observed vaccine effectiveness of $83.2 \%$ in our ecological approach is close to the estimates from outbreaks in the USA which similarly included all levels of severity of varicella.

Vaccination uptake was only assessed in one German federal state; however, vaccination coverage measured for children at school entry is similar in $\mathrm{SH}$ to that in Germany on average [36]. The challenge in estimating vaccination coverage from health insurance data, which provide complete information on all vaccines given, is the generation of an appropriate denominator [20]. The approach to include children with follow-up information up to age 24 months allows defining of a valid denominator. Almost $90 \%$ of children have completed all well-baby check-up visits scheduled within the first 2 years of life. If children without complete well-baby check-up visits were less completely vaccinated, this would account for an overestimation of the vaccination coverage by a maximum of $10 \%$. However, formal assessment of this figure in $\mathrm{SH}$, found an overestimation of only $1 \%$ [37]. As a sensitivity analysis incorporating uncertainty from well-baby check-ups and the extrapolation to other federal states we investigated the following: if using $90 \%$ of the coverage rate observed in Figure 1 as covariate, the resulting estimate for vaccine effectiveness would be $86 \cdot 3 \%$. As a consequence, our reported figure of $83.2 \%$ is conservative with respect to potential overestimation of coverage.

Our time-series modelling makes up an easy to implement alternative to assessing vaccine effectiveness. The similarity of our ecological estimate of varicella vaccine efficacy compared to other approaches can be explained by absence of secular trends and consistent reporting. Lack of large multi-year periodicities is suggested from other epidemiological investigations and modelling approaches [29, 34-36]. Furthermore, there was no specific varicella catch-up vaccination 
programme in Germany. Even though vaccinations have occurred in the 3-4, 5-9 and $>9$ years age groups due to delay between recommended and actual vaccination age [20], the effects can be considered as minor. Finally, ascertainment of cases and vaccination coverage was consistent over time due to the structure of the reporting instruments.

A limitation of the current modelling is that coverage percentage enters the predictor of the log-linear model in linear form. Predictions about the effect of hypothetical $100 \%$ coverage are sensitive to this assumed parametric form. An alternative could be to use more flexible functional forms based on semiparametric penalized spline approaches [38], but in our case such modelling did not indicate strong deviations from the linear form. Furthermore, the multinomial phase 2 model implies a fixed variancecovariance structure of the count data series, which-similar to binomial time series-might be subject to overdispersion [39]. An alternative modelling approach based on a multivariate AR(1) Poisson model with population offsets can be found in Herzog et al. [40]. However, their model does not specifically address the sentinel network situation found in our context and is less flexible with respect to the autoregressive modelling.

It should be noted that standard epidemic models suggest that the number of new cases is proportional to the product of the proportions being infectious and susceptible. Thus, our use of infection pressure in the autoregression represents a pragmatic approximation when no information about the susceptible proportion is available. Theoretically, changes in the susceptible proportions due to vaccination could be reflected by a time-varying autoregression parameter. Furthermore, as herd effects are reflected in the unstratified level 1 model, we implicitly assume that these effects are homogeneous over all age groups.

Finally, given the short period of the German vaccination programme, our analyses of vaccine effectiveness do not take into account that immunity from vaccination might wane over time which would confound our effectiveness estimate. Re-assessment of waning immunity might become necessary, once the programme has been in operation for a longer time.

Altogether, our vaccine effectiveness estimates correspond to immediate effects and thus do not reflect long-term impact. It is well known that vaccination effects can be exaggerated during the initial period of a vaccination programme, e.g. due to 'honeymoon effects' [41] or boosting from natural varicella cases which is expected to decline in the future [42]. Hence our estimates may not be directly comparable with effectiveness based on individual-level data since they are subject to transient herd effects. However, the proposed two-phase multivariate time-series modelling represents a novel view on investigating population-level vaccine effectiveness for an age-stratified population indirectly taking current herd effects into account. With this model, the dynamics using a monthly time resolution were investigated and quantified. Such modelling is especially helpful in case of short time-series when starting monitoring immunization programmes without available prevaccination data.

\section{APPENDIX}

\section{Statistical methodology}

Allow $n_{t}$ to be the number of sentinel network reporting units at time $t ; c_{t}$ the total number of reported varicella cases at this time and $\left(c_{t,<1}, c_{t, 1-2}, c_{t, 3-4}, c_{t, 5-9}\right.$, $\left.c_{t,>9}\right)^{\mathrm{T}}$ a column vector of length 5 containing the number of cases in each of the five age groups. The elements of this vector sum to $c_{t}$. At the first level, our interest is in modelling the response $y_{t}=c_{t} / n_{t}$, i.e. a random variable reflecting the mean number of cases per reporting unit at time $t$. To reflect the fact that the response is non-negative, we transform the response using the natural logarithm and hence model $\log \left(y_{t}\right)$. Now, a time-series model is used to model trend and seasonality:

$\log \left(y_{t}\right)=\beta_{0}+\beta_{1} t+f(t)+\varepsilon_{t}$,

where $t=1,2, \ldots, 42$ reflects time (in months) and $\varepsilon_{t}$ is possibly correlated zero mean Gaussian random variables with variance $\sigma^{2}$. Because a log-transformation is used, all effects should be interpreted in multiplicative fashion, i.e. $\exp \left(\beta_{0}\right)$ corresponds to the base level and $\exp \left(\beta_{1}\right)$ is the factor multiplied on this base level for each additional month. Furthermore, $f(t)$ is a periodical function with period 12 months, e.g. $f(1)=f(13)$. The function $f(t)$ is obtained by combining several sine and cosine terms with different frequencies. An advantage of the above regression formulation is that additional covariates can be easily be added to the model.

The expectation of the response can be calculated as

$\mu_{t}=E\left(y_{t}\right)=\exp \left(\beta_{0}+\beta_{1} t+f(t)+1 / 2 \sigma^{2}\right)$. 
When using, e.g. vaccination coverage instead of time as linear covariate, vaccine effectiveness is defined as $1-\mu_{100} \%$ coverage $/ \mu_{0} \%$ coverage $=1-\exp \left(\beta_{1}\right)^{100}$.

Since our data are of time-series nature, we expected the observations $y_{t}$ to be correlated. Even after modelling trend and seasonality as explained above, we expected remaining correlation to be present within the series. If this additional correlation (i.e. autocorrelation) is not taken into account, the standard errors obtained from the modelling would be too optimistic and hence wrong results about significance will be obtained. To capture this autocorrelation, a $\operatorname{ARMA}(p, q)$ model with $p$ autoregressive (AR) and $q$ moving-average (MA) terms is fitted to the residuals, $\varepsilon_{t}$. This modelling approach is a well-known procedure from time-series analysis for fitting seasonal time-series data [43].

Estimation of the model parameters $\beta_{0}, \beta_{1}, \sigma^{2}$, the parameters of $f(t)$ and the coefficients in the seasonal ARMA model given known values of $p, q$ was performed by standard likelihood methods. The appropriate parameters $p, q$ of the $\operatorname{ARMA}(p, q)$ time-series model were selected according to AIC in all nine models with $p, q$ being either 0,1 or 2 . We also investigated seasonal $\operatorname{ARMA}(p, q)$ models, but as the seasonality is modelled explicitly using $f(t)$, no such additional complexity improved the AIC.

The age-specific dynamics in the mean number of cases per reporting unit is modelled by a two-stage approach. First, the above presented seasonal ARMA model captures the development in the mean number of visits per reporting unit over all age groups. Second, the vector of proportions $\left(p_{t,<1}, p_{t, 1-2}, p_{t, 3-4}\right.$, $\left.p_{t, 5-9}, p_{t,>9}\right)^{\mathrm{T}}$ in age groups at a specific time-point is modelled by multinomial logistic regression using category-specific intercept, trend and period function (see e.g. [39]). Altogether,

Level 1:

$$
\begin{aligned}
y_{t} & =\frac{c_{t}}{n_{t}}=\exp \left(\beta_{0}+\beta_{1} t+f(t)+\varepsilon_{t}\right), \\
\varepsilon_{t} & \sim \operatorname{ARMA}\left(p, q ; \sigma^{2}\right),
\end{aligned}
$$

Level 2:

$$
\begin{aligned}
& \left(c_{t,<\mathbf{1}}, c_{t, \mathbf{1 - 2}}, c_{t, \mathbf{3}-4}, c_{t, \mathbf{5}-\mathbf{9}}, c_{t,>\mathbf{9}}\right)^{\mathrm{T}} \\
& \sim \operatorname{Multinom}\left(c_{t},\left(p_{t,<\mathbf{1}}, p_{t, \mathbf{1 - 2}}, p_{t, \mathbf{3}-\mathbf{4}}, p_{t, \mathbf{5}-\mathbf{9}}, p_{t,>\mathbf{9}}\right)^{\mathrm{T}}\right) .
\end{aligned}
$$

When estimating parameters at level 2 we used the actual observed $c_{t}$ and not the level 1 predicted $c_{t}-$ this makes it possible to fit the two models independently from each other. However, for the later predictions we combined level 1 and level 2 models using the multiplication $y_{t} \times\left(p_{t,<1}, p_{t, 1-2}, p_{t, 3-4}, p_{t, 5-9}\right.$, $\left.p_{t,>9}\right)^{\mathrm{T}}$, where $y_{t}$ is now the predicted value from level 1 and the vector of probabilities is the prediction from level 2. With this combination a time-series model for the mean number of cases per sentinel unit in each age group is obtained. We now calculate

$$
\begin{aligned}
\mu_{t, i} & =E\left(y_{t, i}\right) \\
& =\frac{\exp \left(\gamma_{0, i}+\beta_{0}+\left(\gamma_{1, i}+\beta_{1}\right) \cdot t+f(t)+f_{i}(t)+\frac{1}{2} \sigma^{2}\right)}{\sum_{j \in\{<1,1-2,3-4,5-9,>9\}} \exp \left(\gamma_{0, j}+\gamma_{1, j} t+f_{j}(t)\right)},
\end{aligned}
$$

for $i=<1,1-2,3-4,5-9,>9$, with the $\beta$ values and the $\gamma$ values being the intercept and trend parameters in the level 1 and level 2 models, respectively, $f(t)$ and $f_{i}(t)$ being the harmonic functions, and $\sigma^{2}$ being the variance of the level 1 model error term. Note that for the reference age group (e.g. 1-2 years), the restrictions $\gamma_{0,1-2}=\gamma_{1,1-2}=0$ and $f_{1-2}(t)=0$ for all $t$ applies, i.e. no parameters need to be estimated for this group.

A big advantage of our proposed multivariate twophase model, compared to modelling the mean number of varicella cases separately for each age group, is that it takes into account, that the number of cases summed over the age groups equals the observed total number of cases. However, when considering the dynamics within a single age group in our model, the interpretation of the model parameters is not straightforward. Instead, we report ratios such as $\mu_{13, i} / \mu_{1, i}$ giving the relative reduction within the first year of the sentinel network for a specific age group. Confidence intervals for these reduction factors can be obtained by parametric bootstrap exploiting the asymptotic normality of both the $\beta$ values and the $\gamma$ values in the computation of the respective $\mu_{t, i}$ values. When using vaccination coverage as linear covariate instead of time, the relative reduction $\mu_{100} \%$ coverage, $i / \mu_{0} \%$ coverage, $i$ denotes the relative reduction in the expectation of the response in age group $i$-by comparing the $0 \%$ coverage with the $100 \%$ coverage situation. Again, this can be used to define vaccine effectiveness. In case the harmonic functions of time remain in the predictors of the level 1 and level 2 models, one would typically fix time to some arbitrary value, e.g. $t=0$, for the comparison.

\section{ACKNOWLEDGEMENTS}

We thank the participating sentinel physicians for their data reporting and the German Green Cross for collecting the data in the varicella sentinel. The work 
of the first author was financially supported by the LMUinnovativ project 'Munich Center of Health Sciences'.

\section{DECLARATION OF INTEREST}

The Varicella Sentinel is part of the work of the Measles and Varicella Working Group (Arbeitsgemeinschaft Masern und Varizellen), which is a joint initiative of the Robert Koch Institute and the vaccine manufacturers, GlaxoSmithKline and Sanofi Pasteur MSD, together with the German Green Cross. The manufacturers finance the work of the German Green Cross, which is responsible for the realization of the project. The Robert Koch Institute supplied scientific guidance and does not receive any financial support from the vaccine manufacturers.

\section{REFERENCES}

1. Nguyen HQ, Jumaan AO, Seward JF. Decline in mortality due to varicella after implementation of varicella vaccination in the United States. New England Journal of Medicine 2005; 352: 450-458.

2. Reynolds MA, et al. Epidemiology of varicella hospitalizations in the United States, 1995-2005. Journal of Infectious Diseases 2008; 197 (Suppl. 2): S120-126.

3. Davis MM, Patel MS, Gebremariam A. Decline in varicella-related hospitalizations and expenditures for children and adults after introduction of varicella vaccine in the United States. Pediatrics 2004; 114: 786-792.

4. Seward JF, et al. Varicella disease after introduction of varicella vaccine in the United States, 1995-2000. Journal of the American Medical Association 2002; 287 : 606-611.

5. Seward JF, Marin M, Vazquez M. Varicella vaccine effectiveness in the US vaccination program: a review. Journal of Infectious Diseases 2008; 197 (Suppl. 2): S82-89.

6. Kuter B, et al. Ten year follow-up of healthy children who received one or two injections of varicella vaccine. Pediatric Infectious Disease Journal 2004; 23: 132-137.

7. Gould PL, et al. An outbreak of varicella in elementary school children with two-dose varicella vaccine recipients - Arkansas, 2006. Pediatric Infectious Disease Journal 2009; 28 : 678-681.

8. Nardone A, et al. The comparative sero-epidemiology of varicella zoster virus in 11 countries in the European region. Vaccine 2007; 25: 7866-7872.

9. Heininger U, Seward JF. Varicella. Lancet 2006; 368 : 1365-1376.

10. Siedler A, Arndt $\mathbf{U}$. The impact of the routine varicella vaccination programme on varicella epidemiology in Germany. Eurosurveillance 2010; 15(13): pii $=19530$.
11. Halloran ME, Longini IM, Struchiner CJ. Design and Analysis of Vaccine Studies. New York: Springer, 2010.

12. Halloran ME, et al. Theoretical epidemiologic and morbidity effects of routine varicella immunization of preschool children in the United States. American Journal of Epidemiology 1994; 140: 81-104.

13. Brisson M, et al. Analysis of varicella vaccine breakthrough rates: implications for the effectiveness of immunisation programmes. Vaccine 2000; 18: 2775-2778.

14. Farrington CP. Estimation of vaccine effectiveness using the screening method. International Journal of Epidemiology 1993; 22: 742-746.

15. Anderson RM, Grenfell BT, May RM. Oscillatory fluctuations in the incidence of infectious disease and the impact of vaccination: time series analysis. Journal of Hygiene (London) 1984; 93: 587-608.

16. Grijalva CG, et al. Decline in pneumonia admissions after routine childhood immunisation with pneumococcal conjugate vaccine in the USA: a time-series analysis. Lancet 2007; 369: 1179-1186.

17. Antunes JL, et al. Effectiveness of influenza vaccination and its impact on health inequalities. International Journal of Epidemiology 2007; 36: 1319-1326.

18. Girard DZ. Intervention times series analysis of pertussis vaccination in England and Wales. Health Policy 2000; 54: 13-25.

19. Mullooly JP, et al. Evaluation of the impact of an HMO's varicella vaccination program on incidence of varicella. Vaccine 2004; 22 : 1480-1485.

20. Kalies $\mathbf{H}$, et al. Vaccination coverage in children can be estimated from health insurance data. BMC Public Health 2008; 8: 82.

21. R Development Core Team. $R$ : A Language and Environment for Statistical Computing. Vienna, Austria: R Foundation for Statistical Computing, 2009.

22. Halloran ME, Longini Jr. IM, Struchiner CJ. Design and interpretation of vaccine field studies. Epidemiological Review 1999; 21 : 73-88.

23. Bramley JC, Jones IG. Epidemiology of chickenpox in Scotland: 1981 to 1998. Communicable Disease and Public Health 2000; 3: 282-287.

24. Brisson M, Edmunds WJ, Gay NJ. Varicella vaccination: impact of vaccine efficacy on the epidemiology of VZV. Journal of Medical Virology 2003; 70 (Suppl. 1): S31-37.

25. Ferguson NM, Anderson RM, Garnett GP. Mass vaccination to control chickenpox: the influence of zoster. Proceedings of the National Academy of Sciences USA 1996; 93: 7231-7235.

26. Garnett GP, Grenfell BT. The epidemiology of varicella-zoster virus infections: a mathematical model. Epidemiology and Infection 1992; 108: 495511.

27. Marin M, Meissner HC, Seward JF. Varicella prevention in the United States: a review of successes and challenges. Pediatrics 2008; 122: E744-E751.

28. Quian J, et al. Impact of universal varicella vaccination on 1-year-olds in Uruguay: 1997-2005. Archives of Diseases in Childhood 2008; 93: 845-850. 
29. Guris D, et al. Changing varicella epidemiology in active surveillance sites-United States, 1995-2005. Journal of Infectious Diseases 2008; 197 (Suppl. 2): S71-75.

30. Bayer O, et al. Metaanalysis of vaccine effectiveness in varicella outbreaks. Vaccine 2007 ; 25 : 6655-6660.

31. Chaves SS, et al. Loss of vaccine-induced immunity to varicella over time. New England Journal of Medicine 2007; 356: 1121-1129.

32. Spackova M, et al. Comparative varicella vaccine effectiveness during outbreaks in day-care centres. Vaccine 2010; 28: 686-691.

33. Clements DA, et al. Postlicensure study of varicella vaccine effectiveness in a day-care setting. Pediatric Infectious Disease Journal 1999; 18: 1047-1050.

34. Seward JF, et al. Contagiousness of varicella in vaccinated cases: a household contact study. Journal of the American Medical Association 2004; 292: 704-708.

35. Vazquez M, et al. Effectiveness over time of varicella vaccine. Journal of the American Medical Association 2004; 291 : 851-855.

36. Anon. Vaccine coverage at school health examinations in Germany, 2007 [in German]. Epidemiologisches Bulletin 2009; 16: 143-145.

37. Bader HM, Ludwig M. Vaccine coverage from association of SHI physicians billing: regional determination of immunization from birth until the age of 24 months 2003-2007 in the state of Schleswig-Holstein/Northern Germany. In: Abstracts of the 1st National Vaccination Conference, 5-7 March 2009, pp. 22-23 (http://www. nationale-impfkonferenz.de/media/Abstractband_5_03_ 09_.pdf), 2009.

38. Fahrmeir L, Tutz G. Multivariate Statistical Modelling Based on Generalized Linear Models, 2nd edn. New York: Springer, 2001.

39. Mebane WR, Sekhon JS. Robust estimation and outlier detection for overdispersed multinomial models of count data. American Journal of Political Science 2004; 48: 392-411.

40. Herzog SA, Paul M, Held L. Heterogeneity in vaccination rates explains the size and occurence of measles epidemics in German surveillance data. Epidemiology and Infection. Published online: 12 July 2010. doi:10.1017/S0950268810001664.

41. McLean AR, Anderson RM. Measles in developing countries. Part II. The predicted impact of mass vaccination. Epidemiology and Infection 1988; 100: 419-442.

42. Goldman GS. The case against varicella vaccination. International Journal of Toxicology 2006; 25: 313-317.

43. Shumway RH, Stoffer DS. Time Series Analysis and its Applications. New York: Springer, 2000. 